\title{
Rhetorical Compositions for Controlled Natural Languages
}

\author{
Andrew Potter \\ Sentar, Incorporated, 315 Wynn Drive, Suite 1 \\ Huntsville, Alabama, USA 35805 \\ andrew.potter@sentar.com
}

\begin{abstract}
Logic-based controlled natural languages usually provide some facility for compositional representation, minimally including sentence level coordination and sometimes subordination. These forms of compositional representation are useful for expressing short passages of a few sentences, but compared to natural language they offer only a limited range of expression, and they are unwieldy for expressing longer passages. This paper describes a method for bringing more naturalness to controlled natural languages. This approach defines a model for representing compositional structures found in natural language, such as Antithesis, Concession, and Unless in a way that is both rhetorically expressive and logically reducible. This is demonstrated through a series of examples using both natural and controlled natural language. The paper then describes a set of intercompositional rules of inference that can be used to reason about the interrelationships between compositional structures, facilitating the discovery and assessment of supportive and conflicting relationships among them. This ability to represent and discover interrelationships among discourse representation structures could be useful for developing applications that must not only follow a set of rules of inference, but reason about the rules themselves.
\end{abstract}

Keywords: Controlled Natural Language, Rhetorical Structure Theory, Argumentation, Knowledge Representation, Logic, Explanation Aware Computing

\section{Introduction}

Logic-based controlled natural languages usually provide some facility for compositional representation. Most well known among these, ACE and PENG define discourse representation structures that support sentence level coordination and subordination $[1,2]$, and CLCE, CPL, and E2V support sentence level coordination [3-5]. These forms of compositional representation are useful for expressing short passages of a few sentences, but relative to natural language they offer only a limited range of expression, and they soon become unwieldy for expressing longer passages. New techniques are needed for representing compositions in a way that is both rhetorically expressive and logically reducible.

In this paper we describe an approach to addressing this need. We first describe the theoretical foundations for our approach, based on Rhetorical Structure Theory 
and the Toulmin model of argumentation. We then show that complex compositional relations found in natural language can be reduced to propositional logic, and we show how these relations can be applied to controlled natural languages to produce more expressive texts. Because the structures are reducible to logical expressions, it is possible to use them to construct highly expressive knowledge representations. Next we introduce a set of intercompositional rules of inference that can be used to reason about the chaining interrelationships among these compositional structures, facilitating the discovery and evaluation of supportive and conflicting relationships among them. Finally, we conclude with a discussion of the significance of these observations, as well as some directions for future research.

\section{Theoretical Basis}

The approach presented here defines a mapping between Rhetorical Structure Theory [6] and the Toulmin model of argumentation [7]. RST supplies rhetorical relations and structural constraints used to define expressive composititonal representations in a logically reducible manner. The Toulmin model provides an argumentative framework for applying a set of rhetorical rules of inference. Just as the Toulmin model uses a warrant to establish a link between a ground and a claim, RST establishes links between a nucleus and its satellites. An example of this mapping is shown in Fig. 1, where two statements are related using the RST Evidence relation, such that the satellite corresponds to the argumentative ground and the nucleus corresponds to the claim. By mapping Toulmin with RST, we can say that an RST relation is used to characterize a warrant in terms of its specific relationship between its claim and its ground, and moreover, that any claim may have any number of grounds, just as in RST a nucleus may have multiple satellites.

More formally, we can say that this model defines warrants, spans, statements, relations, and inference rules, such that a warrant establishes a set of links between a nucleus and zero or more satellites, as shown in Fig. 2. The nucleus and its satellites are represented as spans. A span consists of a CNL statement and, in the case of satellites, the satellite's relation to its nucleus. In argumentative terms, the nucleus corresponds to a claim, and the satellites correspond to grounds. Each satellite (or ground) links to the nucleus (or claim) by means of a rhetorical relation. Note that a statement can be either a elementary discourse unit or it can be another warrant. This permits the construction of nested structures.

For the purposes of this analysis, RST relations are treated as either inferential or synthetic. An inferential relation is one whose satellite has an argumentative, causal, or logical implication for the nucleus, such that if the satellite is instantiated, the nucleus may be inferred. Examples of inferential relations include Antithesis, Condition, Concession, Evidence, Unless, and the various causal relations defined by RST. In contrast to inferential relations, synthetic relations are purely informational, so that the satellite tends to provide information relating to the nucleus, but the satellite is not material to the nucleus. Examples of synthetic relations are Background, Circumstance, Elaboration, and Summary. Intercompositional inference rules are defined for characterizing the interrelationships among argumentative structures; for example, one instance of Evidence will substantiate another if its claim 
(nucleus) unifies with the ground (satellite) of the other. These intercompositional rules of inference and their significance for knowledge representation will be explored in detail later in this paper.

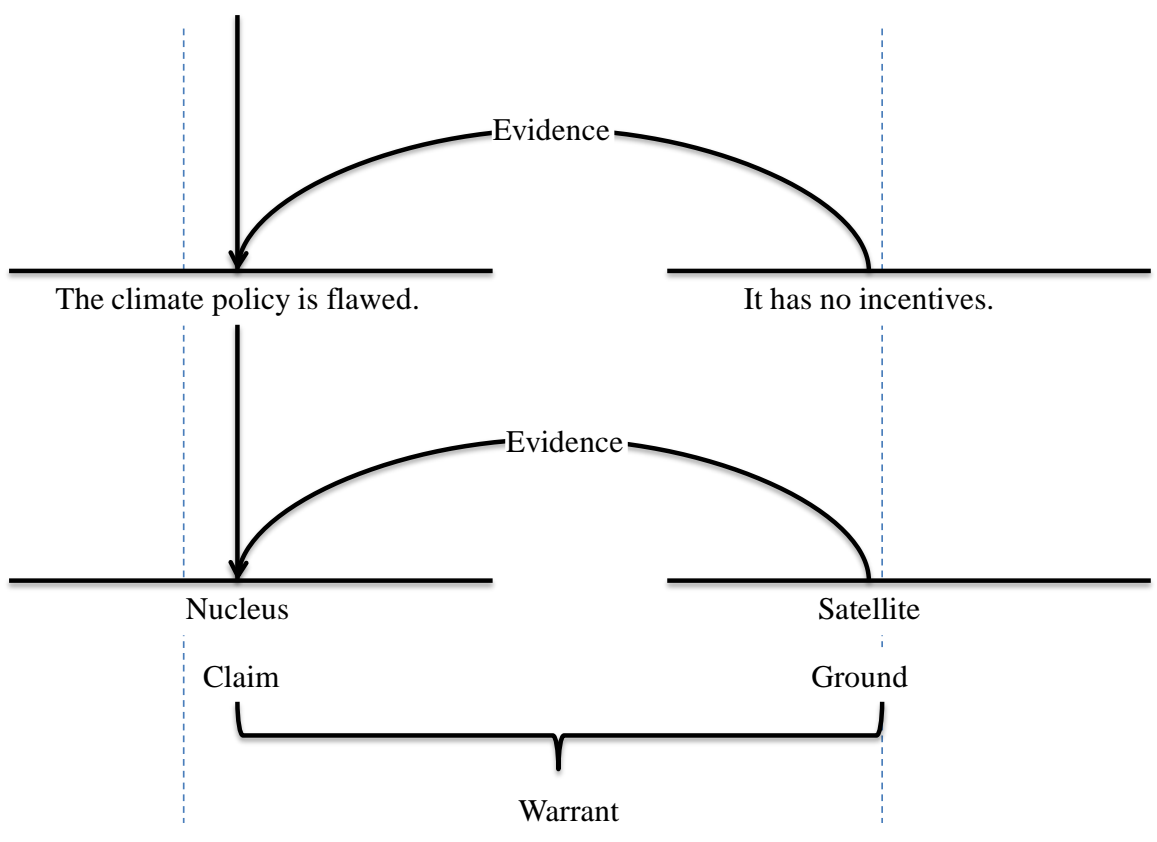

A policy that lacks an incentive is flawed.

Fig. 1. Toulmin RST mapping shows the correspondence between an inferential rhetorical relation and a warrant.

\section{The Logic of Rhetorical Relations}

Having outlined the underlying theoretical considerations, we can make this more concrete by showing through examples how rhetorical relations can be mapped into propositional logic. In the following, we give examples of key inferential rhetorical relations using natural language. Once we have established that this approach is applicable to natural language, we can then take the next step, of applying the same techniques to logic-based controlled natural languages to produce more expressive compositional structures.

\subsection{Antithesis \& Concession}

Antithesis and Concession are closely related rhetorical relations. Antithesis is used to increase the reader's positive regard for the nucleus by introducing incompatible 
information in the satellite that casts the nucleus in a more positive light. Similar to Antithesis, the Concession relation seeks to increase the reader's positive regard for the nucleus. However, it accomplishes this not by introducing incompatible information in the satellite, but by acknowledging an apparent in compatibility between the nucleus and satellite in such a way that the effect is that the reader's positive regard for the nucleus is increased. So the difference between the two is subtle. Indeed, Stede [8] argued that the difference is so subtle that perhaps there is not one. He claimed that Antithesis and Concession are nearly identical, except that Antithesis is more general since, unlike Concession, it places no constraints in the satellite. However, there is another important difference-namely that in the Antithesis relation there is an incompatibility between the satellite and nucleus, whereas with Concession the incompatibility is merely apparent.



Fig. 2. The Reasoning Model defines a mapping between RST and the Toulmin model.

An analysis of the underlying logic of Antithesis and Concession suggests that while the given definitions of these two relations are similar, there are important differences in logical structure. This can be seen clearly through examination of a few examples:

1) What engenders respect is not the particular outcome that a legal scholar arrives at but, rather, the intellectual rigor and honesty with which he or she arrives at a decision. 
This example consists of two segments, and they are clearly intended as incompatible. And clearly the writer (or speaker) has a preference for the latter of the two. Hence the relation is one of Antithesis. Now if the rhetorical structure can be mapped to an argument structure, then the argument ground and claim should correspond to the satellite and nucleus, and we need to supply a plausible warrant for binding the two. The claim is that respect is engendered by the intellectual rigor and honesty with which a legal scholar arrives at a decision; the ground is that respect is not engendered by the particular outcome the scholar arrives at. So how does the claim follow from the ground? Here is a logical interpretation of Antithesis:

$$
(\sim(\mathrm{g} \equiv \mathrm{c}) \cdot \sim \mathrm{g}) \supset \mathrm{C}
$$

That is, the ground $\mathrm{g}$ and claim $\mathrm{C}$ are incompatible, and since not $\mathrm{g}, \mathrm{C}$. That this is a plausible interpretation can be seen by revisiting the original text. Having set up the incompatibility between these two means of engendering respect, we negate one of them, hence implying the other. To this extent we establish positive regard for the nucleus by means of indicating negative regard for the satellite. However, we cannot expect that every instance of Antithesis will include a satellite stated with explicit negative quality:

2) I'd rather lose an election than see my country lose a war.

—John McCain

While it might seem unlikely that any politician would under any circumstances prefer to lose an election, it is this expectation that gives the statement its rhetorical punch. The speaker identifies two incompatible situations, and makes clear, given the choice, his preference between the two. Though there is no explicit negation in the satellite, the speaker's positive regard for the nucleus is clearly indicated. Thompson and Mann [9] found that in natural language (English) the use of the contrastive conjunction Rather often signals Antithesis.

Concession increases positive regard for the nucleus by acknowledging an apparent incompatibility, but by recognizing that this incompatibility is only apparent, the writer increases reader's positive regard for the situation presented in nucleus. If the satellite and nucleus were really incompatible, then the nucleus might be cast in doubt:

3) And I thought we could muddle through without the governor's personal chef — although I've got to admit that sometimes my kids sure miss her.

-Sarah Palin

There is no incompatibility between muddling through without the chef and admitting that the kids sure missed her. That is, since $\mathrm{g}$ does not imply that $\mathrm{C}$ is false, C must be true:

$$
\sim(\mathrm{g} \supset \sim \mathrm{C}) \supset \mathrm{C}
$$


As a generalized structure, such patterns cannot be escalated to prescriptive argument schema, because not all instantiations will be true [10]. But as a persuasive pattern of reasoning, Concession occurs frequently in natural language, and therefore is a structure of interest for controlled natural languages. The argument trades on the lack of incompatibility between the satellite and nucleus. This is quite different from Antithesis, where the argument trades on the recognition of their incompatibility.

\subsection{Evidence and Other Modus Ponens Relations}

In the Evidence relation, comprehension of the satellite increases the reader's belief of the Nucleus. That is, the satellite provides evidence for the nucleus. Here is an example:

4) There is far too much arresting of people going on. Not just Skip [Henry Louis] Gates - a couple of weeks ago, some poor woman in Montauk was arrested in her bathing suit, marched off the beach and charged with a felony for forging a beach parking sticker.

-Nora Ephron

The claim is that "far too much arresting of people going on," and in support of this claim, the writer cites a particular example as evidence. The logical structure of the Evidence relation, as well as many other inferential relations, is modus ponens.

$$
((g \supset c) \cdot g) \supset c
$$

Other inferential relations following this pattern are Condition, Justify, Motivation, Enablement, Means, and Purpose, as well as the causal relations, NonvolitionalCause, Nonvolitional-Result, Volitional-Cause, and Volitional-Result. These relations all follow the familiar modus ponens logical structure. Note that with Motivation and Enablement, the nuclear claim is not an assertion of fact, but an action. Here is an example of Motivation:

5) We do, quite literally, possess the means to destroy all of mankind. We must seek to do all we can to ensure that nuclear weapons will never again be used.

-John McCain

In this example the claim is a call to action motivated by the threat posed by the current availability of nuclear weapons. Just as with Evidence, where the reader might not believe the nucleus without the satellite, but with Motivation, the satellite increases the reader's desire to perform action presented in nucleus. 


\subsection{Unless}

In Rhetorical Structure Theory, the definition of Unless states that the Nucleus is realized provided that the Satellite is not. In argumentative terms, this states that negation of the ground implies the claim:

$$
\sim \mathrm{g} \supset \mathrm{C}
$$

One might reasonably take a step further here and say that the RST definition also entails that if the ground is not negated, then the claim is negated:

$$
g \supset \sim \mathrm{C}
$$

Or, in other words

$$
\sim(\mathrm{g} \equiv \mathrm{c})
$$

which corresponds to what has been referred to in logic as the strong sense of Unless [11]. And clearly the connective is sometimes used in this way:

6) Unless it's a life or death emergency, no credible doctor is going perform surgery on a child for any reason without a parent's permission.

-Richard Shelby

Surgery on a child without the parent's permission will be performed if and only if there is life or death emergency. But in the weak sense, which is the more common use of Unless, a negative ground implies the claim, but a positive ground does not necessarily negate the ground. Here is an example:

7) And unless we free ourselves from a dependence on these fossil fuels and chart a new course on energy in this country, we are condemning future generations to global catastrophe.

-Barack Obama

Given that the options for global catastrophe are numerous, charting a new course on energy will not guarantee a future free of global catastrophe; it will eliminate one possible catastrophic scenario. The presence of the cue word "unless" makes an RST encoding of the relation Unless difficult to resist, even when the weak sense is intended.

If the Unless relation is to be used in a CNL, it will be necessary to pick one interpretation and stick with it. Since the weak sense is the more common usage in natural language, it seems reasonable that this would be the preferred interpretation. But there is another reason for adopting the weak sense of Unless. Unless can be combined with other arguments to serve as a Toulmin qualifier. In the Toulmin model, a qualifier is used to indicate that the linkage between the ground and the 
claim may be subject to exception. That is, the Unless relation limits the warrant, as in this example:

8) When the Federal Reserve extends a loan or purchases a security, this automatically adds reserves to the banking system unless the Fed undertakes an offsetting reserve draining operation.

In this example, the warrant is that activities such as extending loans put money into the system. The draining operation is cited as a circumstance when the warrant would not apply. So in the absence of the exception the warrant holds:

$$
\sim \mathrm{g} \supset \mathrm{w}
$$

But it seems unreasonable to suggest that the negative ground and the warrant are materially equivalent, as would be required by the strong sense. The difficulty becomes apparent when we expand the warrant,

$$
\sim g_{q} \supset\left(g_{w} \supset c_{w}\right)
$$

since $\left(g_{w} \supset c_{w}\right)$ will hold anytime $c_{w}$, regardless of whether $g_{w}$. In our example, the strong sense would require that a draining operation had been undertaken any time no addition to the banking systems was made. The use of Unless as a qualifier brings us to a further observation. We need not be limited to relations between elementary discourse units; indeed we can construct relations between units and structures to create more complex structures, and we can even relate one structure to another. Further, these structures need not necessarily be known during composition, but may be discovered and integrated during runtime reasoning. Used in this way, Unless is an example of a particular intercompositional rule of inference call dissociation, to be discussed in detail later in this paper.

\section{CNL and the Logic of Rhetorical Relations}

We have now seen how inferential rhetorical relations can be mapped into propositional logic. This indicates that the resulting compositions are not "merely rhetorical," but that they are also shorthand for complex logical expressions. To the extent that this mapping is possible in natural language, it may also be a desirable feature for controlled natural languages. To explore this, we will construct examples using simple ACE statements as elements and add rhetorical relations for sentence level coordination. We can then compare these compositions with logically equivalent $\mathrm{ACE}$ compositional representations. The rhetorical relations used here are Concession, Antithesis, Causality, and Unless.

\subsection{Concession}

As discussed earlier, Concession appeals to an apparent incompatibility between ground and claim, such that the claim is strengthened by acknowledgement of the 
ground. That is, it is not the case that the ground denies the claim, and therefore the claim is seen as more likely. To illustrate this in CNL, we start with two statements:

9) Some individuals believe that the climate problem is a scientific hoax.

10) The climate problem is not a scientific hoax.

and develop a Concession relation between the two, signaled by the connective although:

11) Although some individuals believe that the climate problem is a scientific hoax, the climate problem is not a scientific hoax.

Without the Concession relation, the CNL equivalent of this would be:

12) If it is false that if some individuals believe that the climate problem is a scientific hoax then it is false that the climate problem is not a scientific hoax then the climate problem is not a scientific hoax.

That is, that some individuals believe that the climate problem is a scientific hoax does not mean that it is a hoax, and that lends support to the claim that it is not. Although the reasoning underlying the Concession relation may be argumentum ad ignorantium, support for compositional representations employing although would nevertheless give writers a useful tool for making statements that are currently impracticable with the current CNL technology.

\subsection{Antithesis}

Antithesis appeals to an incompatibility between ground and claim, but unlike Concession, the incompatibility is claimed to be real rather than apparent:

13) The climate problem is a scientific hoax.

14) The climate is a critical problem.

An antithetical relationship can be established when we negate the first statement and apply rather as a connective between the two:

15) The climate problem is not a scientific hoax, rather the climate is a critical problem.

Using the logical definition given earlier (1), without support for antithetical compositions, the example can be written in CNL as:

16) If it is false that if the climate problem is a scientific hoax then the climate is a critical problem, and it is false that if the climate is a critical problem then the climate problem is a scientific hoax, and it is false that the climate problem is a scientific hoax then the climate is a critical problem. 
Making this readable requires more than fixing up the commas. The statements 15) and 16) are logical equivalent, but the negated material equivalence embedded in Antithesis is more readily expressed using rather rather than nested conditionals. While natural language affords numerous ways to construct antithetical expressions [9] , the ability to use rather for sentence level coordination would clearly lend power to any CNL.

\subsection{Causality}

Rhetorical structure theory defines a variety of volitional and non-volitional causal relations, but for our purposes we need only one composite causal relation, which we may call Because. Using the causal relation we can construct the following compositional relation from a set of simple statements:

17) All scientists know that the climate is a problem, because the atmosphere is polluted, and the sea-levels are higher, and the average temperatures are higher.

which conveys a sense of causality not expressible without the Because relation. At best, we can create compound conditional, such as the following:

18) If the atmosphere is polluted, and the sea-levels are higher, and the average temperatures are higher then all scientists know that the climate is a problem.

Although the gain in readability achieved through use of the causal relation is less than that of relations such as Concession and Antithesis, by identifying the causal nature of the relationship between ground and claim, we do provide richer expressiveness than is possible relying on the if-then structure.

\subsection{Unless}

As noted earlier, the Unless relation, as used in natural language, indicates that the claim of an argument is realized provided that the ground is not. Here is an example using two CNL statements:

19) Unless all emissions are controlled soon, the climate problem is hopeless.

with the logical equivalent of

20) If all emissions are not controlled soon then the climate problem is hopeless.

The weak form of Unless is used here, as is preferable for CNL, in order to support the its use as a Toulmin qualifier, as discussed earlier. The qualifier indicates that the linkage between the argument ground and claim may be subject to exception. The 
weak form allows this to happen, while having no effect on the argument when there is no exception. We can construct a CNL example of Unless as a Toulmin qualifier from the following elements:

21) No rich countries lead.

22) Some developing countries make no improvements.

23) The developing countries experience a global-warming disaster.

First we construct the argument:

24) If no rich countries lead then some developing countries make no improvements.

And then we qualify it:

25) If no rich countries lead then some developing countries make no improvements, unless they experience a global-warming disaster.

According to this argument, some developing countries require the leadership of rich countries in order to undertake self-improvement on their own, but if things get bad enough, they may initiate these improvements on their own. Here is how this can be represented in CNL without the Unless relation:

26) If some developing countries experience a global-warming disaster then it is false that if no rich countries lead then the developing countries make no improvements.

As with the other examples we have seen here, while the statements are logically equivalent, the naturalness of the content is overshadowed by the need for explicit logical controls. As will be developed in the discussion of intercompositional rules of inference, the availability of the Unless relation would provide a powerful tool for specifying the terms of dissociation within an argument.

\section{Intercompositional Rules of Inference}

Thus far we have focused on how the logic of rhetorical relations could be used to write more expressive compositional representations in CNL. However, taking the view of these compositions as warrants suggests some interesting possibilitiesnamely that we might be able to define rules that enable automated reasoning about the structures, thus identifying the interrelationships among them. These rules, called intercompositional rules of inference, are identified in in Table 1. Basically, these rules identify the kinds of inferential chaining that may occur among compositions. An intercompositional inference occurs when the nucleus, satellite, or warrant of one structure can be unified with the nucleus, satellite, or warrant of some other structure. In the Substantiation rule, the claim of one argument is used as the ground of another; this contrasts with Undercut, where the claim of one argument is incompatible with 
the ground of another. With Concomitance, two arguments share the same ground to establish distinct claims, whereas with Confusion, the grounds of two arguments are incompatible. With Rebuttal, the claims of two arguments are incompatible, while with Convergence, two grounds converge upon a shared claim. The Backing rule states that the claim of one argument substantiates the warrant of another, whereas with Dissociation, the claim of one argument disputes the warrant of another. In the following discussion, we take a closer look at some of these rules.

Table 1. Intercompositional Rules of Inference

\begin{tabular}{ll}
\hline \multicolumn{1}{c}{ Rule } & Definition \\
\hline Substantiation & The claim of one argument is used as the ground of another \\
Rebuttal & The claims of two arguments are incompatible \\
Backing & An argument substantiates the warrant of another \\
Undercut & The claim of one argument is incompatible with the ground of another \\
Dissociation & The claim of one argument disputes the warrant of another \\
Convergence & Two arguments lead to the same claim \\
Concomitance & Two arguments use the same ground to establish distinct claims \\
Confusion & The grounds of two arguments are incompatible \\
\hline
\end{tabular}

\subsection{Substantiation}

Substantiation occurs when the claim of one argument unifies with the ground of another. Substantiating arguments may be chained to one another through a claimground linkage. Here is a straightforward example using simple conditional arguments:

27) If the climate problem is not a scientific hoax then the climate is a critical problem.

28) If the climate problem is a critical problem, then it is necessary that all governments act soon.

This seems simple enough. However, since the Substantiation rule applies whenever the claim of one argument unifies with the ground of another, it is possible to use other relations, such as Concession and Antithesis, to allow for more complex inferences based on the Substantiation rule. In the following example, the ground that some individuals believe that the climate problem is a scientific hoax ultimately substantiates claim that the climate is a critical problem:

29) Although some individuals believe that the climate problem is a scientific hoax, the climate problem is not a scientific hoax.

30) The climate problem is not a scientific hoax, rather the climate is a critical problem. 


\subsection{Backing}

Backing is similar to Substantiation, except that the claim being substantiated is a warrant, consisting of both ground and claim. That is, an argumentative relation is substantiated, not just its ground or its claim. Here is an example of a Backing rule:

31) It is true that if no rich countries lead then some developing countries experience a global-warming disaster, because they lack the necessary motivation.

In this argument, the claim consists of the conditional statement it is true that if no rich countries lead some developing countries experience a global-warming disaster, and the ground is because some developing countries lack the necessary resources. That is, the strength of the conditional is grounded by its backing. Using structures such as this, a knowledge base can achieve added explanatory power. In a fully developed knowledge base, an instance of the Backing rule could be the claim of some further argumentation.

\subsection{Dissociation}

If we wish to have the means to explain why an argument should be accepted, then we should also provide for when it should not. Thus the Backing rule is counter-balanced by Dissociation. We have already seen some examples of Dissociation in the discussion of the Unless relation as a Toulmin qualifier. The effect of Unless is to dissociate the ground from claim, thereby limiting the warrant, for example:

32) If no rich countries lead then some developing countries make no improvements, unless they experience a global-warming disaster.

By the Dissociation rule, the warrant that the absence of improvement among developing countries could be attributable to a lack of leadership from richer countries would be inapplicable when circumstances included a global-warming disaster. In other words, any instantiation of the rule

33) If no rich countries lead then some developing countries make no improvements.

is weakened by the qualifier, if the qualifier is also instantiated. Thus the Unless relation makes it possible to make this determination at runtime. The use of the Unless relation maps easily to the dissociation rule, as it is the classic Toulmin exception. However, other relations can be used to implement Dissociation. Here is an example using Antithesis:

34) It is not true that if the climate problem is a critical problem, then it is necessary that all governments act soon, rather nobody has a solution. 
The Concession relation can be used similarly:

35) Although it is not true that if the climate problem is a critical problem, then it is necessary that all governments act soon, nobody has a solution.

Thus there are several ways to dissociate a warrant. The Unless relation limits a warrant by identifying possible exceptions to it, Concession weakens it by suggesting that it might not be to the point, and Antithesis rejects it outright.

\subsection{Convergence}

Convergence is a more difficult case. The Convergence rule applies when multiple arguments lead to the same claim. In natural language, Convergence is difficult to analyze logically because its structure supports multiple semantics. As noted by Walton [12], in a purely convergent argument, multiple premises support a conclusion, but they do so independently. Yet there are also arguments of identical structure where the premises combine to support the conclusion. These Walton refers to as linked arguments. In a linked argument, neither premise alone is sufficient to establish the conclusion. In contrast, a merely convergent argument is actually multiple arguments which share the same conclusion. In natural discourse, it is not always easy to distinguish linked from convergent arguments. Consider this example,

36) All scientists know that the climate is a problem, because the atmosphere is polluted, and the sea-levels are higher, and the average temperatures are higher.

In this argument, the conclusion is supported by several conditions. But how should this be interpreted? Would scientists still know that the climate is a problem if one or two of the three conditions were not met? It might be the case that any one of these conditions would be sufficient to reach the conclusion. So it is possible that this example is a case of argumentative accrual, or what Walton calls an evidenceaccumulating argument [12].

But if there are evidence-accumulating arguments, then there might also be evidence- dissipating arguments, where the arguments in a convergence cancel one another out - as in the example used by Prakken [13], where one argument uses rain as a reason not to go jogging and the argument other uses heat as a reason not to go jogging, but with the result that the two arguments converge upon a single claim. It is important to note that this interpretation relies on ontological insight —one must understand something about jogging in order to detect the cancellation effect.

So there are four possible interpretations of a convergence structure: the argument could be convergent, it could be linked, it could be evidence-accumulating, or it could be evidence dissipating. For CNL, it would be possible to signal whether a structure is linked, accumulating, dissipating, or merely convergent through the use of cue words. For example, a term such as furthermore could be used to indicate an evidence-accumulating argument. However, this approach does not fully address the 
problem, because convergent structures may also be discovered at runtime [14]. For example, suppose we have a knowledge base containing the following arguments:

37) All scientists know that the climate is a problem, because the atmosphere is polluted.

38) All scientists know that the climate is a problem, because the sea-levels are higher.

39) All scientists know that the climate is a problem, because the average temperatures are higher.

Using the Convergence rule, we can infer a convergent structure. Since the statements occur independently, we may also infer that they are not linked. But there is no structural basis for supposing that the structure is evidence-accumulating or evidence-dissipating. Thus, the minimal case, that convergent structures discovered a posteriori are simple convergences seems reasonable - in other words, the rule at work here is a convergence rule, and not a linked-argument, evidence-accumulating, evidence-dissipating rule. The most that can be inferred at runtime is a convergence relationship.

\section{Conclusion}

In this paper, we have examined composite discourse structures in natural language and used this to develop new possibilities for expressiveness in controlled natural languages. By showing that rhetorical relations are reducible to propositional logic, we suggest that they could be incorporated into CNLs to provide writers with enhanced resources for expressiveness while assuring that any resulting expressions could be parsed and interpreted by a reasoning system. Enabling CNL writers to incorporate such composite expressions into their knowledge representations would enable them to achieve added explanatory power.

Intercompositional rules of inference open the possibility of extending the scope of compositional activity beyond authorship to include runtime discovery of supportive and conflicting relationships among compositional structures. This ability to represent and discover interrelationships within and among structures in an explicit way could prove useful for developing applications that not only follow a set of rules of inference, but reason about the rules themselves. This suggests the possibility of introducing techniques for defeasible reasoning into controlled natural languages. For example the current approach could be extended to include the use of qualifiers and qualification ratios [15] for use in representing argument defeasibility, with resulting qualification values propagating through networks of rhetorical structures. It also seems possible that some rhetorical relations may be construed as stronger than others; for example, arguments based on causality may be stronger than arguments based on Concession. Additional research is needed to understand the full ramifications and utility of these possibilities. 


\section{References}

1.Fuchs, N.E., Kaljurand, K., Kuhn, T.: Discourse representation structures for ACE 6.0. Department of Informatics, University of Zurich, Zurich (2008)

2.Schwitter, R.: English as a formal specification language. Proceedings of the 13th International Workshop on Database and Expert Systems Applications (2002) 228-232

3.Pratt-Hartmann, I.: A two-variable fragment of English. Journal of Logic, Language and Information 12 (2003) 13-45

4.Sowa, J.: Common logic controlled English (2007)

5.Clark, P., Harrison, P., Jenkins, T., Thompson, J., Wojcik, R.: Acquiring and using world knowledge using a restricted subset of English. FLAIRS (2005) 506-511

6.Mann, W.C., Thompson, S.A.: Rhetorical structure theory: Towards a functional theory of text organization. Text 8 (1988) 243-281

7.Toulmin, S.E.: The uses of argument. Cambridge University Press, Cambridge, UK (1958)

8.Stede, M.: Disambiguating rhetorical structure Research on Language \& Computation 6 (2006) 311-332

9.Thompson, S.A., Mann, W.C.: Antithesis: A study in clause combining and discourse structure. In: Steele, R., Threadgold, T. (eds.): Language Topics: Essays in Honour of Michael Halliday, Volume II. John Benjamins, Amsterdam (1987) 359-381

10.Walton, D.: Explanations and arguments based on practical reasoning. Proceedings of Workshop W10: Explanation-Aware Computing, Twenty-First International Joint Conference on Artificial Intelligence, Pasadena (2009) 72-83

11.Hardegree, G.: Symbolic logic: A first course Mcgraw-Hill (1999)

12.Walton, D.N.: Argument structure: A pragmatic theory. University of Toronto Press, Toronto, Canada (1996)

13.Prakken, H.: A Study of Accrual of Arguments, with Applications to evidential reasoning. The Tenth International Conference on Artificial Intelligence and Law, Proceedings of the Conference, June 6-11, 2005. ACM, New York (2005) 85-94

14.Potter, A.: Linked and convergent structures in discourse-based reasoning. In: RothBerghofer, T., Schulz, S., Bahls, D., Leake, D.B. (eds.): Proceedings of the 3rd International Explanation Aware Computing Workshop (ExaCt 2008), Patras, Greece (2008) 72-83

15.Fox, J., Das, S.: Artificial intelligence in hazardous applications. AAAI Press, Menlo Park, CA (2000) 